\title{
The polar belts of prominence occurence as an indicator of the solar magnetic field reversal
}

\author{
Teodor Pintér, Milan Rybanský and Ivan Dorotovič
}

Slovak Central Observatory, Komárňanská 134, SK-94701 Hurbanovo, Slovak Republic email: teodor.pinter@suh.sk, rybansky.milan@gmail.com, ivan.dorotovic@suh.sk

\begin{abstract}
The global magnetic field of the Sun is the determining parameter of spreading the solar wind in the interplanetary space. The global field changes the polarity synchronically with the cycle of solar activity. The interesting indicator of the polarity change are the occurence so-called polar belts of the prominences. The article shows the performance of these belts on observational work from 1975 to 2009 . A coordinated effort is suggested for the compilation of data from different observers following the method described by Rušin et al., 1988 .
\end{abstract}

Keywords. Prominence occurence, polar belts, solar magnetic field reversal

\section{Introduction}

Although the magnetic field of the Sun is measured for a long time, the existence of polar field was often questioned until the 1950s. An argument for the existence of such field was mainly based on observations of polar coronal rays during total solar eclipses. However, it failed to be confirmed by measurements. Svalgaard et al. (1978) published, for the first time, a methodology for calculating the polar fields using measurements of background magnetic field of the Sun from the heliographic latitudes $\pm 55^{\circ}$ to the poles. These measurements have been carried out at Stanford since 1975. The resulting data are published at http://wso.stanford.edu.

The maximum magnetic field strength occurs at the poles during the minimum of the solar activity cycle and reaches values about $0.1-0.2 \mathrm{mT}$. It is peculiar, that in the last three cycles the absolute value of magnetic field strength continues to decrease. Another interesting fact is that the polarity reversal of the poloidal magnetic field, according to the data does not occur at the same time. Therefore, during a certain period of time the Sun has the same polarity on the both poles.

\section{Initial data, method of processing, and results}

In this contribution we would like to point out a fact that a concomitant event of the polarity reversal of the magnetic field of the Sun is an 'arrival' of polar branches of prominences to the solar poles. It seems that prominences occur randomly across the surface of the Sun. This applies only to heliographic latitudes of $\pm 40^{\circ}$, and even this is not valid everywhere. In higher latitudes is clearly observable the so called polar branch of prominences (Fig. 1 and 2).

The Figures 1 and 2 are drawn on the basis of the catalogue of prominences compiled according the observations in the coronal station of the Astronomical Institute at Lomnický Štít (Rušin et al., 1988 and subsequent supplements up to 2009). Each marker in the figure depicts observation of one prominence at a given time and at a given latitude. 


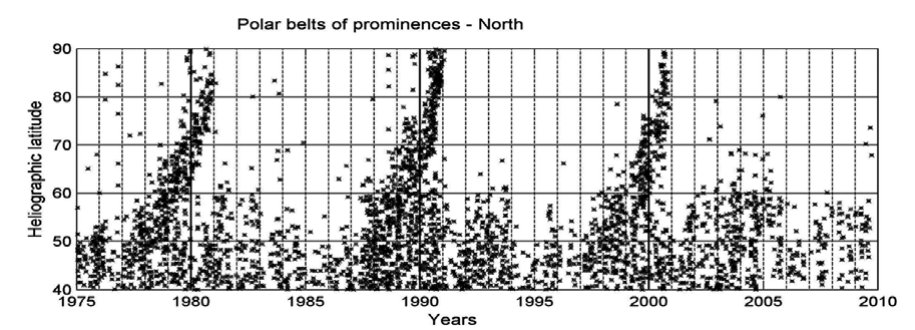

Figure 1. Polar branches of prominences near the North Pole of the Sun.

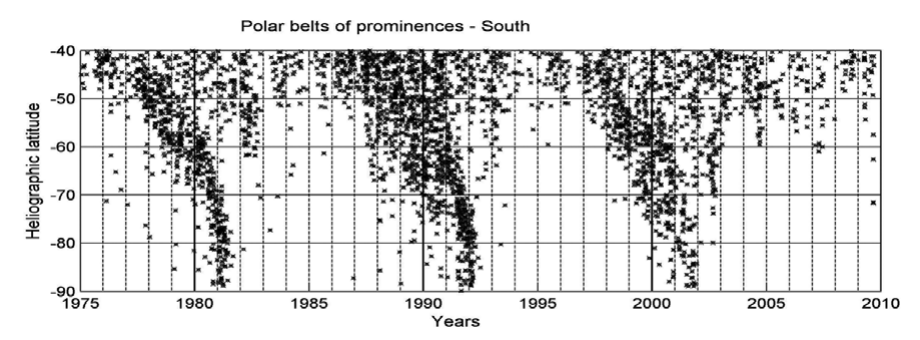

Figure 2. Polar branches of prominences near the South Pole of the Sun.

Only prominences the area of which is larger than 50 units are considered. Area is in the catalogue indicated in units of $1^{\circ} \times 1^{\prime \prime}$, the range in positional angles is given in degrees, and the height of prominences in arc seconds. Catalogue in the electronic form can be requested via emailaddressed to suh@suh.sk.

The method of observation and hence the catalogue itself was created upon the initiative of Dr. Gnevyshev (Russia) and therein are published observations from above mentioned observatory from 1967 to 2009. From the images we can see that apart from the period around the polarity reversal prominences (of the given kind) did not occur at latitudes above $65^{\circ}$. This fact can be used to predict the time of the polarity reversal.

Prominences reached the pole:

1981.2 $\mathrm{N}$ and 1981.2 $\mathrm{S}$ in the 21st cycle; $1990.8 \mathrm{~N}$ and 1992.0 $\mathrm{S}$ in the 22nd cycle; $2000.7 \mathrm{~N}$ and 2001.8 $\mathrm{S}$ in the 23rd cycle;
Polarity reversal occured:

$1979.9 \mathrm{~N}$ and $1980.5 \mathrm{~S}$

$1990.3 \mathrm{~N}$ and $1991.3 \mathrm{~S}$

$2000.5 \mathrm{~N}$ and 2000.0 S.

\section{Conclusions}

From these data one would assume that the polarity reversal occurs at a time when the polar branches of prominences reach a latitude around $\pm 70^{\circ}$. Observation of prominences is performed at many professional and amateur institutes. The authors believe that it would be appropriate to consolidate the method of processing of observations and to publish them electronically in a standardized form by one institute. This contribution uses observations only from one observatory, thus there are big gaps caused by the weather. Inspite of that also from such observations can be possible to contribute to reveal a mechanism of solar cycle which is not well understood, yet.

\section{References}

Rušin, V., Rybanský, M., Dermendjiev V., \& Stavrev K. Y. 1988, Contrib. Astron. Obs. Skalnaté Pleso, 17, 63

Svalgaard, L., Duvall, T. L. jr., \& Scherrer, P. H. 1978, Solar Phys., 58, 225 